\title{
PREVALENCE OF SUBSTANCE USE AMONG RURAL HIGH SCHOOL STUDENTS IN LIMPOPO PROVINCE, SOUTH AFRICA
}

\author{
Hans E. Onya* \\ Sovenga, South Africa \\ Alan J. Flisher \\ University of Cape Town \\ Cape Town, South Africa
}

School of Public Health, University of the Limpopo

\begin{abstract}
This study documents the prevalence rates for use of cigarette, alcohol, methylated spirit, cannabis, mandrax and cannabis together, glue or thinners among rural high school students in Mankweng, Limpopo Province, South Africa. A multistage sampling procedure produced a sample of 1600 students in grades 9 and 11 who completed a self-administered questionnaire. The prevalence rates for previous mouth (recent) use of alcohol, cigarettes, cannabis glue and spirits were $6.4 \%, 10.5 \%$, $1.4 \%, 1.2 \%$ and $0,8 \%$ respectively. For all substances, males had higher prevalence rates than females. Developing alcohol and drug programme for high school students that are more gender specific may improve the effectiveness of intervention efforts at high schools.
\end{abstract}

KEY WORDS: substance use, rural high school students, South Africa

\section{INTRODUCTION}

Globally, cannabis is the most widely consumed illicit drug, with an estimated 114 million people using cannabis annually. Although cannabis is not the principal problem drug of abuse in Europe, the Americas, Australia or Asia, it is the main problem drug of abuse in Africa (UN, 2000). The results of the analysis of the changes in cigarette use among high school students in the United States indicated that although 1) the prevalence of life-time cigarette use was stable among high school students during 1990s and 2) the prevalence of both lifetime and current frequent cigarette use increased into the $1990 \mathrm{~s}$, these behaviours had declined significantly by 2003 . The average age of the onset of alcohol use among high school students in the United States in 1995 was 14 years (Escobedo et al, 1995). There has been a trend of decreasing alcohol consumption in most developed countries, in many developing countries, levels of alcohol consumption have increased in recent years (Flisher, 2003; Gurije, 2000; Obot, Karuri \& Ibanga, 2003; Parry, 2000; Peden, van Der Spuy, Smith \& Bantz, 2000; Rehm \& Room, 2003).

Studies addressing alcohol, tobacco and other drugs (ATOD) use from Africa have focused on questions such as prevalence rates, opinions, influence of the media on use and interventions such

\footnotetext{
* Corresponding Author: Hans Onya, Health Promotion Unit, School of Public Health, University of Limpopo, Sovenga 0727, South Africa: Tel: +27 - 15 - 263 6129; Fax: + 27 - 15 - 2636432 e-mail: ho7@mweb.co.za
} 
as impact of peer led education. Some of these studies have demonstrated a change in drinking pattern in that traditional and socioculturally integrated modes of drinking have been replaced by socially disruptive drinking, and rates have increased substantially among adolescents and women (Adelakan \& Ndom, 1997; Department of Health, 2002; Eide \& Acuda 1995; Flisher, Parry, Evans, Muller \& Lombard 1998, 2003; Henry J. Kaiser family Foundation, 2001; Karim, Mohammed, Ahmed \& Mohammed 1998; Khan \& Arnott, 1996; Kuria, 1996; May, Brook, Gossage, Oxford, Adnams, Jones, Robinson \& Viljoen, 2000; Parry, 1994; Peltzer \& Cherian, 2000; Rocha-Silva, De Miranda \& Erasmus, 1996) Other studies have concluded that among adolescents, boys are generally speaking at higher risk for substance use than girls (Escobedo, Chorba, \& Wexmeiler, 1995; Flisher et al 2003; Peltzer, Cherian \& Cherian, 1999; Rosenberg, Kasl, \& Berberian, 1974; Smart \& Fejer, 1971; Wildgoose, 1997). Age correlates positively with alcohol use and abuse among adolescents (Escobedo, et al, 1995; Wildgoose, 1997).

In Limpopo Province of South Africa where this study was undertaken, Peltzer (1999) found that among urban secondary school pupils, the majority of both boys and girls first used any substance at the age of 16 years or less. Use of tobacco and inhalants was particularly likely to commence at such an early age. One other study in the area that provided data on substance use by adolescents in Limpopo was that conducted by Madu \& Malta in 2003. The study reported prevalence rates of $12.0 \%$, for substance use, (cigarette smoking 10.6\%, and alcohol use 39.1\%). Cannabis was the most used substance and the mean age for first time users was 14.89 years, 14.54 years, 15.25 years for substance, cigarette and alcohol respectively. These findings apply to the Capricorn district as a whole and included urban, private and semiurban, farm and a few rural schools combined and had small sample size $(\mathrm{n}=62, \mathrm{n}=32, \mathrm{n}=19$ for drug use, cigarette smoking and alcohol use).

There has been no published study with a representative sample that has documented the prevalence of substance use among exclusively rural black high school students of specific ethnic group (The Pedi ethnic group) living in a specific district in South Africa. This current study used similar methodology that was used in the Cape Town study referred to above (Flisher et al, 2003) and had a representative sample of high school students of Pedi origin. The main aim was to document substance use behaviour of these students and to determine whether this differ in any way from "Black" high school students that were studied in Cape Town and high school student in some other parts of subSaharan Africa. There are reasons to believe that the understanding of the pattern of behaviour of a specific group of people towards substance use or abuse, will better inform the development of culture- and area-specific interventions on substances use or abuse that will be effective. This study aims to fill this gap.

\section{METHOD}

\section{Study Population and Sampling}

This study population comprised of all students attending high schools in Mankweng Dristict in Limpopo Province. The province has a population of 5.4 million. Of these, $97.1 \%$ are black, $2.7 \%$ are white, and $0.1 \%$ coloured and Indian/Asian each; $45.7 \%$ of them are male; and $16.1 \%$ are teenagers. Many of the population live under poor economic 
conditions and have a low level of access to health facilities (Health Systems Trust and the Department of Health, 1997; Statistics South Africa, 2000).

There are 63 high schools in Mankweng District, all of which are public. Twenty schools were randomly selected from the 63 schools for the study, such that the probability of selection of a school was directly proportional to the number of students in the school. A total of 80 students were selected from each school; 40 were randomly selected from each of grade nine and grade eleven. According to the school records, only a total number of $25(1.5 \%)$ students absented themselves from school on the day of the research without any genuine reason or permission. A total of 1600 students took part in the study.

\section{Instrument}

The instrument in this study was previously used in surveys of adolescent substance use in urban settings (Flisher, Ziervogel, Chalton \& Robertson, 1993; Flisher et al, 2003). Students provided selfreport data on a variety of health-related risk behaviours, including substance use. The questionnaire included 15 items on the socioeconomic background. In addition the questionnaire contained items about whether the student had used various substances. The questions involving substances referred to use of alcohol; drinking methylated spirit; smoking a whole cigarette; smoking "dagga"/marijuana; smoking Mandrax (methaqualone) and dagga together; sniffing glue or thinners; using crack cocaine; using ecstasy; using any illegal drug such as heroine, stimulants, halucinationgenics such as LSD, Nexus, MMDA, injected drugs; and using any other drug. There was also an item that asked how old students were when they used the substance for the first time.
Students were asked if they had used a fictitious substance (Derbisol), and the eight students that responded positively to this item were excluded.

Bilingual speakers translated the instrument into Northern Sotho/Pedi (the local language) and back translated the Northern Sotho version into English. In addition, the appropriateness of the questions for rural South African adolescents and young adults was established through focus group discussion with similar aged students at a randomly selected school from the same administrative province (i.e. Limpopo Province). The focus group discussions confirmed that these drugs are the ones used in Mankweng with the exemption of crack cocaine; ecstasy; illegal drug such as heroine, stimulants, halucinationgenics such as LSD, Nexus, MMDA and injected drugs.

\section{Procedure}

The instrument was administered during a normal class period by two research assistants. The research assistants, both of whom have Northern Sotho as home language, administered the measure under conditions approximating those of examinations. To preserve the privacy of the students, the teachers were not present during the administration of the questionnaires. Anonymity and confidentiality were stressed. Each question was read to the students and the students answered each question simultaneously. Permission for the students to take part in the study was granted by the Department of Education and Culture of Limpopo Province.

\section{Analysis}

Each substance had 3 questions associated with it. The first question was whether the substance had ever been used before (lifetime use), followed by 
questions on recent use and current (i.e. whether it had been used in the past year and in the past month respectively). If the first question involving lifetime use was omitted, and the subsequent question has a positive response indicating that the student had used the substance in the previous year or month, we took this as indicating that the student has in fact used the substance in the previous year or month.

Observations with missing values were excluded from the calculation of that particular prevalence rate, except in the case where a student answered negatively to the first question and left out the 2 recent use questions. In these cases negative answers for the following 2 questions were assumed to be negative even though they contained missing values. Those who had given a positive response for recent use over the past month along with a negative response (or missing value) for the past year question, had their value for the past year question changed to be positive i.e. it is not possible to have used a substance during the past month but not during the past year.

All prevalence rates were expressed as percentages. If the lower bound of a particular $95 \%$ confidence interval is negative it has been presented as a zero since a negative prevalence rate is meaningless. We compared the results for each gender (within each grade) and for each grade (within each gender). In comparing two groups, if the 95\% CI's do not overlap, there is a significant $(\mathrm{p}<0.025)$ difference between the groups. If the CI's overlapped but not to the extent that the point estimate of one group is contained within the CI of the other group, there is a significant $(p<0.05)$ difference between the groups. If they overlap to the extent that the point estimate of one group is contained within the CI of the other group, we cannot draw any conclusions as to whether there is a significant difference between the groups (Fisher, 1959).

\section{RESULTS}

The total sample size was 1600 . Of these, $740(46.2 \%)$ were male, $795(49.6 \%)$ were female and $65(4.2 \%)$ did not report their gender. The age range was 11-29 years (mean age 16.4 years; Standard deviation $=2.79)$. The ages of 663 $(41.4 \%)$ were between 1 and 15 years, $790(49.4 \%)$ were between 16 and -20 years, $117(7.3 \%)$ between 21 and 25 years; and $8(0.5 \%)$ were 25 years of age or older. 22 (1.4) did not report their ages. There were 800 respondents each in grade 9 and grade 11. Of the total sample size of 1600. A further 22 did not report their gender and were also excluded. The analysis was confined to 1570 students. Table 1 indicates lifetime, past year and past mouth (recent) prevalence rates (with their $95 \%$ CIs) respectively of most commonly used substances. For lifetime, past year and past month use alcohol, cigarettes, dagga, glue and spirits were, in descending order, the most prevalent substances used.

Prevalence rates for Ecstasy, crack, Mandrax, illegal drug such as heroine, stimulants, hallucinogenics such as LSD, Nexus, MMDA, injected drugs and other drugs (salicyte analgesics, stimulants, antibiotics, hypo-sedatives, and organic solvents) were generally very low (below $1 \%$ ) or not reported at all. For all substances, males had higher prevalence rates than females. Alcohol was the most used drug for both boys $(52,7 \%)$ and girls $(13,4 \%)$ in grade 11 . For both grades 9 and 11 combined, the prevalence rates for alcohol are 36, 2\% for males and 10,9\% for female. There was a trend for the rates to increase from grade 9 to grade 11 for all the substances. No student reported ever using mandrax, crack and ecstasy. 
Table 1. Prevalence rates by gender and grade among Grade 9 and 11 students in Mankweng, Limpopo Province $(n=1570)$

\begin{tabular}{|c|c|c|c|c|c|}
\hline & & & GRADE 9 & GRADE 11 & Total \\
\hline \multirow[t]{12}{*}{ BOYS } & \multirow[t]{3}{*}{ Cigarettes } & Lifetime & $6,9(4,4-9,4)$ & $28,5(20,1-37)$ & $18,2(12,6-23,7)$ \\
\hline & & Past year & $4,5(2,5-6,6)$ & $23,1(16-30,1)$ & $14,2(9,4-18,9)$ \\
\hline & & Past month & $4,1(2,1-6,1)$ & $21,3(14-28,7)$ & $12,9(8,2-17,5)$ \\
\hline & \multirow[t]{3}{*}{ Alcohol } & Lifetime & $17,5(9,2-25,8)$ & $52,7(45,2-60,2)$ & $36,2(30,9-41,5)$ \\
\hline & & Past year & $8,5(2,4-14,6)$ & $36,9(31,1-42,7)$ & $23,5(19,1-27,9)$ \\
\hline & & Past month & $6,1(2,5-9,8)$ & $30,1(23,1-37,1)$ & $18(13,4-22,5)$ \\
\hline & \multirow[t]{3}{*}{ Spirits } & Lifetime & $0,9(0-2)$ & $3,4(1,2-5,5)$ & $2,2(1-3,4)$ \\
\hline & & Past year & $0,5(0-1,6)$ & $1,1(0-2,2)$ & $0,9(0,2-1,6)$ \\
\hline & & Past month & $0.5(0-1,6)$ & $1,2(0-2,3)$ & $0,9(0,2-1,6)$ \\
\hline & \multirow[t]{3}{*}{ Cannabis } & Lifetime & $1(0-2,4)$ & $10,5(5,7-15,4)$ & $6,1(3,1-9,1)$ \\
\hline & & Past year & $0,5(0-1,3)$ & $6,2(3,3-9,2)$ & $3,6(2-5,2)$ \\
\hline & & Past month & $0,3(0-0,8)$ & $5(2,4-7,7)$ & $2,7(1,4-4,1)$ \\
\hline \multirow[t]{12}{*}{ GIRLS } & \multirow[t]{3}{*}{ Cigarettes } & Lifetime & $2,1(0,5-3,7)$ & $1,7(0,5-2,9)$ & $1,9(1-2,8)$ \\
\hline & & Past year & $0,5(0-1,3)$ & $0,5(0-1,3)$ & $0,5(0-1)$ \\
\hline & & Past month & $0,5(0-1,3)$ & $0.5(0-1,3)$ & $0,5(0-1)$ \\
\hline & \multirow[t]{3}{*}{ Alcohol } & Lifetime & $8,1(3,9-12,3)$ & $13,4(9,9-16,9)$ & $10,9(8,2-13,7)$ \\
\hline & & Past year & $3,7(1-6,5)$ & $7(4,3-9,7)$ & $5,5(3,6-7,4)$ \\
\hline & & Past month & $2,7(0,9-4,5)$ & $5,3(3,1-7,5)$ & $4,1(2,7-5,5)$ \\
\hline & \multirow[t]{3}{*}{ Spirits } & Lifetime & $1,4(0-2,8)$ & $1,7(0,4-2,9)$ & $1,5(0,5-2,6)$ \\
\hline & & Past year & $0,8(0-2)$ & $0,8(0-1,6)$ & $0,8(0,1-1,4)$ \\
\hline & & Past month & $0,8(0-2)$ & $0,8(0-1,6)$ & $0,8(0,1-1,4)$ \\
\hline & \multirow[t]{3}{*}{ Cannabis } & Lifetime & $1(0-2,3)$ & $0,8(0-1,8)$ & $0,9(0-1,8)$ \\
\hline & & Past year & $0,5(0-1,4)$ & $0,4(0-1,1)$ & $0,4(0-1,3)$ \\
\hline & & Past month & $0,5(0-1,4)$ & 0 & $0,2(0-0,7)$ \\
\hline
\end{tabular}

For students in both grades 9 and 11 , the CIs for boys and girls did not overlap in any case. There was a trend for the rates to increase from grade 9 to grade 11 for boys, with the $95 \%$ CIs not overlapping in all the cases. For girls, the CIs for the two grades overlapped in all cases. The CIs for boys and girls did not overlap for alcohol, cigarette and cannabis for grade 11 recent users. The prevalence rate for the use of sprits and cannabis for girls in both grades 9 and 11 were below $1 \%$ for girls and the CIs for both grades overlapped.

\section{DISCUSSION}

This study found a lower prevalence of substance use among black Pedi high school students compared to other studies.
Prevalence rates for ecstasy, crack cocaine, heroine, mandrax and glue or thinners were not reported at all for the total population. Boys used more substances than girls. Boys' substance use seems to increase with grade. For girls, the reverse is the case expect for alcohol that increases with grade as well. Girls are less likely to use spirit, "dagga"/marijuana, cigarette and even alcohol than boys regardless of grade.

Specifically, the prevalence rates of various substances (cigarette, alcohol, methylated spirit; dagga mandrax and dagga together glue or thinners, crack cocaine, ecstasy, illegal drug such as heroine, stimulants, halucinationgenics such as LSD, Nexus, MMDA, injected drugs) use were lower in this study compared to other studies (Flisher et al, 
1993, 2003; Madu \& Matla, 2003; Obot, Karuri, \& Ibanga, 2003; Peltzer et al., 1999; UN, 2000). The lower rates in rural schools in Limpopo Province may be accounted for by the fact that most of the inhabitants are likely to be affected by the traditional African values that discourage the use of drugs by children. According to these values, it would be unmannered, uncultured, and a sign of parental irresponsibility for a typical black African Child to use drugs (Madu $\&$ Matla, 2003). They may be less likely to be influenced by other cultural and economic, media and globalization factors.

This study found a lower past month prevalence rate for alcohol use among girls than that of boys in the same grades as was found among black high school students in Cape Town (Flisher et al, 2003). It was also lower than that reported for adolescents in Urban, Periurban and rural high schools combined, in Limpopo (Madu 2003), and for grade 11 urban high school students in Limpopo (Peltzer, 1999). The rates were eleven times higher than that of white female students in grade 11 in Cape Town compared to female students in grade 11 in rural Limpopo high Schools. However, the findings of low prevalence rates for black females in Cape Town (Flisher et. al, 1998, 2003), South Africa (Rocha - Silva, 1996) and Limpopo (Peltzer, 1999; Peltzer \& Phaswane, 1999; Madu 2003) compared with black males is in line with this study. Culture, access, opportunities, role modelling and other community constructs may account for this trend as there are great disparities in these conditions in urban areas like Cape Town compared to those in rural areas such as Mankweng.

In this study, the prevalence rates of cigarettes use were higher for boys than girls in grades 9 and 11. These low prevalence rates for girls may lie in the character of Mankweng (the study site) which is entirely inhabited by blacks, and substance use, especially cigarette smoking, is culturally a predominantly male activity and often not acceptable among females (Madu \& Matla, 2003). Females that smoke cigarette is generally regarded to be wayward and some even relate them to sex work .The same explanation goes for cannabis smoking among grade 11 . No rates were reported for grade 9 boys and girls for both dagga and glue use. However, the thirteen years of democracy in South Africa has resulted in policy and legislative reforms that seem to be changing the social and economic circumstances of rural communities including Mankweng in Limpopo province. The effect is that Mankwneg is becoming urbanised, poverty is being alleviated, more families are being empowered economically, basic infrastructure is improving, more and more adolescents are attending schools, and women and children are becoming aware of their "rights". At the same time, development efforts appear to be eroding traditional values. For example, the impact of legislation and social roles of traditional leaders in the preservation of traditional African culture is competing seriously with the western culture. Western cultures seem to be influencing traditional ways of the life of people in Mankweng. If this trend continues, adolescent risk behaviours, including the use of substances, is likely to increase and the current prevalence rates of substance use among adolescents in Limpopo province may increase in the near future.

The prevalence rates for Mandrax, crack and ecstasy were generally low (below 1\%) for both males and females in the population. This is not in line with the 
findings reported among urban, periurban and rural adolescents studied together in Limpopo (Madu and Malta 2003) which found a prevalence rates of crack cocaine of $8.1 \%(\mathrm{n}=435)$ for boys and $8,3 \%$ for girls and Peltzer, (1999) that reported prevalence rates of crack of $8.1 \%$ for boys and $6,7 \%$ for girls $(n=191)$. These differences may be because both studies included urban high schools and had small and unrepresentative samples of high schools students in Mankweng or the Province as a whole. For example, schools were excluded because the roads leading to them were not accessible (Madu \& Malta 2003, page 406). In other words, the sample was not representative of the population in which they were drawn. This unrepresentativeness may limit generalisation of their findings in Mankweng. The present study was carried on in only rural schools in Mankweng and had a representative sample of black Northern Sotho speaking students. Another possible reason for this may be related to the fact that many families in the Limpopo rural $(94.6 \%)$ communities live under very poor economic and medical conditions (Health Systems Trust and the Department of Health (1997). As a result many children have no money or access to alcohol and other substances. In addition, the black population $(97.1 \%)$ in Limpopo Province are to a great extent, sturdy holders of their culture and tradition. In the Pedi culture, use of any of the substances mentioned above by adolescents is an unacceptable antisocial behaviour and as a result, adolescents are not expected to use substances. This cultural attribute combined with poverty may have prevented substance peddlers from urban areas from finding market for their business.

Unlike high school students in Cape Town (Flisher et al 2003) this sample did not report taking drugs like ecstasy or crack. This is consistent with the finding of Peltzer and Cherian (1999). A reason for this may be that these substances are more readily available in major urban centres like Cape Town and Durban than in rural Mankweng. The finding that, generally, male students had higher rates of substance use than female students is congruent with other studies.

We are inclined to belief that the pattern of substance use for males and females among high school students of the Pedi ethnic group in Limpopo Province of South Africa could be described to be similar to that of their black counterparts that were studied in Cape Town study (Flisher, 2003) and other studies from Sub-Saharan African countries for alcohol, tobacco, cannabis, mandrax and glue. However, the prevalence rates seem to be lower for all the substances mentioned above. In other words Pedi adolescents attending rural high school appear to use less substance than their urban counterparts in other parts of Sub-Saharan Africa.

This study has some limitations. It relied exclusively on self-reports which are subject to distortion and social desirability effects. The design effect was not taken into account in the analysis. This sample was completely homogeneous in terms of race, and schooling is high school students aged 11-29 years. Given this, external validity of these findings may limit generalisation to Limpopo province as a whole. A proportion of those who tend to exaggerate their substance use may have been excluded from the study by the omission of those (eight students) who answered positively to use of a fictitious drug. The sample included the Pedi ethnic group only and did not account for the two other black ethic groups (Venda and Shangan) that make up the 
$97 \%$ of black people living in the Limpopo Province. Excluding these two distinct ethnic groups may have produced different prevalence rates of substances use among black high school students in Limpopo Province. The alcohol and other drugs users were not asked to indicate the quantity of alcohol or drugs taken or time of consumption in a day. There was no way of assessing underreporting of substance use. The above limitations should be noted by researchers.

\section{CONCLUSION}

To achieve maximum impact on substance use prevention efforts among high school students, educational efforts should not attempt single, nation-wide programmes. Developing substance sue programme for high school students that are more gender specific may improve the effectiveness of intervention efforts at high schools. This study supports a call for carefully targeted gender-responsive interventions. This could decrease future substance use problems and set the stage for this high school generation to provide a healthier environment for future generations

\section{REFERENCES}

Adelekan, M. L. \& Ndom, R. J. (1997). Trends in prevalence and pattern of substance use among secondary school pupils in Ilorin, Nigeria. West African Medical Journal, 16(3): 157-64.

El-Fatih Idris A. Karim, Hamid Mannan Mohamed, Mohamed Ibrahim Mohamed, Ali Farah Ahmed and Sana Abdel Azim Mohammed (1998). Drug use among prisoners in three main prisons in Khartoum, Sudan. Eastern Mediterranean Health Journal 4 (1), 122127

Escobedo, L.G. Chorba, T.L. \& Waxweiler, R. (1995). Patterns of alcohol use and the risk of drinking among US high school students. American Journal of Public Health, 85, 976- 978.

Fisher, R. A. (1959). Statistical methods and scientific inference $\left(2^{\text {nd }}\right.$ ed.). Edinburgh, Scotland: Oliver and Boyd.

Flisher A. J., Parry C. D. H., Evans J. Muller M. and Lombard C. (2003). Substance Use by Adolescents in Cape Town: Prevalence and Correlates. Journal of Adolescent Health, 32, 58-65.

Flisher, A., Parry C. Evans J. Lombard C. \& Muller M. (1998). Prevalence rates of alcohol, tobacco and other drug (ATOD) use among Cape Town students in grades 8 and 11. Paper presented at the $4^{\text {th }}$ Annual Congress of the Psychological Society of South Africa, Cape Town, 11 September.

Flisher A. J., Ziervogel, C. F., Chalton, D. O., Leger, P. H.,\& Robertson, B. A. (1993). Risk-taking behaviour of Cape Peninsular high school students: Parts IV. Alcohol use. South African Medical Journal, 83, 480-482.

Health System Trust and the Department of Health (1997). Health care in the Northern Province. Durban: Kwik Kopy Printing,

Henry J. Kaiser Family Foundation. (2001). Portrait of young South Africa. Johannesburg:.

Kuria, M., W. (1996). Drug abuse among urban as compared to rural secondary schools students in Kenya: a short communication. East African Medical Journal, 73(5), 339.

May, P.A. Brooke L. Gossage, J.P. Oxford, J. Adnams C. Jones, K. L Robinson. L. \& Viljoen, D. (2000). Epidemiology of fetal alcohol syndrome in a South Africa community in the Western Cape Province. American Journal of Public Health, 90, 1905-1912.

Madu, S. N. \& Matla, M. P. (2003). Correlations for Perceived Family Environmental Factors with Substance use among adolescents in South Africa. Psychological report, 92, 403-415

Obot, I. S., Karuri, G. S., \& Ibanga A. J. (2003). Substance use and other risky behaviours of secondary school students in a Nigerian urban area. African Journal 
of Drug \& Alcohol Studies, 2 (1\&2), 5765.

Parry, C.D.H. (1994) Prevalence of alcohol misuse in South Africa: a review of empirical research. MRC Urbanisation \& Health Newsletter, 21, 33-38.

Parry, C. D. H, Bhana, A., Pluddemann, A., Myers, B, Siegfried, N, Morojele, N. K., Flisher, A. J. and Kazel, N. J. (2002). The South African Community Epidemiology Network on Drug Use (SACENDU): description, findings (1997-99) and policy implications. Addiction, 97, 69976

Peltzer K. \& Cherian L, (2000) Substance use among urban and rural secondary school pupils in South Africa. Psychological Reports, 87, 582-584.

Peltzer, K, Cherian, V. I., and Cherian L. (1999). Substance use among urban secondary school pupils in the Northern Province, South Africa. Southern African Journal of Child and adolescent mental health Vol 11 No 1.

Peltzer, K., \& Phaswane, N. (1999). Substance use among South African university students: a quantitative and qualitative study. South African HealthInfo.
Penden, M., Van Der Spury, J., Smith, P., \& Bautz, P. (2000). Substance abuse and trauma in Cape Town. South African medical Journal, 90(3), 251-255.

Rehm, J., \& Room, R. (2003). The global burden of disease 2000 for alcohol, tobacco and illicit drugs. Presentation based on World Health Report 2002 at Fremantle, Australia.

Rosenberg J.S Kasl, S.V. \& Berberian, R.M. (1974) Sex differences in adolescent drug use. Addictive Diseases: An International Journal, 1, 73-96.

Rocha-Silva L. de Miranda S. \& Erasmus, R. (1996). Alcohol, tobacco and other drug use among South Africa youth. Human Sciences Research Council Pretoria:

Smart, R.G. \& Fejer, D. (1971) Recent trends in illicit drug use among adolescents. Canada's Mental Health Supplement, 68, 12.

Statistics South Africa.( 2000) Statistics in brief. Pretoria: Central Statistics.

Wildgoose, J. A. (1997) The relationship of risk factors on Bahamian adolescents' alcohol use and alcohol-related problems: a social learning approach. Unpublished dissertation, Howard University, Washington, DC. 